\title{
Identification of effector genes from the phytopathogenic Oomycete Plasmopara viticola through the analysis of gene expression in germinated zoospores
}

\author{
Pere MESTRE ${ }^{a, b, *}$, Marie-Christine PIRON ${ }^{a, b}$, Didier MERDINOGLU ${ }^{a, b}$ \\ ${ }^{\mathrm{a}}$ INRA, UMR 1131 Santé de la Vigne et Qualité du Vin, F-68000 Colmar, France \\ buniversité de Strasbourg, UMR 1131 Santé de la Vigne et Qualité du Vin, F-68000 Colmar, France
}

\section{A R T I C L E I N F O}

Article history:

Received 14 November 2011

Received in revised form

27 April 2012

Accepted 30 April 2012

Available online 15 May 2012

Corresponding Editor:

Meritxell Riquelme

Keywords:

Avirulence genes

Disease resistance

Grapevine

Oomycete

Secretome

\begin{abstract}
A B S T R A C T
Grapevine downy mildew caused by the Oomycete Plasmopara viticola is one of the most important diseases affecting Vitis spp. The current strategy of control relies on chemical fungicides. An alternative to the use of fungicides is using downy mildew resistant varieties, which is cost-effective and environmentally friendly. Knowledge about the genetic basis of the resistance to P. viticola has progressed in the recent years, but little data are available about $P$. viticola genetics, in particular concerning the nature of its avirulence genes. Identifying pathogen effectors as putative avirulence genes is a necessary step in order to understand the biology of the interaction. It is also important in order to select the most efficient combination of resistance genes in a strategy of pyramiding. On the basis of knowledge from other Oomycetes, $P$. viticola effectors can be identified by using a candidate gene strategy based on data mining of genomic resources. In this paper we describe the development of Expressed Sequence Tags (ESTs) from P. viticola by creating a cDNA library from in vitro germinated zoospores and the sequencing of 1543 clones. We present $563 \mathrm{pu}-$ tative nuclear P. viticola unigenes. Sequence analysis reveals 54 ESTs from putative secreted hydrolytic enzymes and effectors, showing the suitability of this material for the analysis of the P. viticola secretome and identification of effector genes. Next generation sequencing of cDNA from in vitro germinated zoospores should result in the identification of numerous candidate avirulence genes in the grapevine/downy mildew interaction.
\end{abstract}

(c) 2012 The British Mycological Society. Published by Elsevier Ltd. All rights reserved.

\section{Introduction}

Grapevine downy mildew caused by the biotrophic Oomycete Plasmopara viticola (Berk. \& Curt. ex. de Bary) is one of the most important diseases affecting Vitis spp. (Viennot-Bourgin 1949). Plasmopara viticola attacks all grapevine green tissues, including leaves, berries, tendrils, and shoots. In the absence of control it causes defoliation as well as drying of berries and stalk, leading to important losses of yield (Gessler et al. 2011). Plasmopara viticola is a heterothallic diploid whose life cycle consists of alternate sexual and asexual phases. The asexual phase is polycyclic and runs over the period of grapevine vegetative growth, while the sexual phase is responsible for the production of overwintering oospores, which represent the primary inoculum for the next season. A typical asexual cycle starts with a zoospore encysting next to stomata and then producing

\footnotetext{
* Corresponding author. UMR 1131 Santé de la Vigne et Qualité du Vin, 28 rue de Herrlisheim BP 20507, 68021 Colmar Cedex, France. Tel.: +33389224947; fax: +33389224933.

E-mail address: pere.mestre@colmar.inra.fr 1878-6146/\$ - see front matter ( 2012 The British Mycological Society. Published by Elsevier Ltd. All rights reserved. doi:10.1016/j.funbio.2012.04.016
} 
a germinative tube that penetrates inside the leaf through the stomata. After forming a vesicle in the substomatic cavity, the pathogen grows in the form of intercellular hyphae, producing haustoria to obtain nutrients from the plant. After a variable incubation time, which depends on environmental conditions, sporulation takes place on the abaxial side of the leaf via emission of sporangiophores through stomata. Release of sporangia, where zoospores are formed, marks the start of a new cycle.

Grapevine downy mildew is currently controlled by chemical fungicides. Using downy mildew resistant varieties is a cost-effective and environmentally friendly alternative (Bisson et al. 2002). However, since all Vitis vinifera cultivars producing quality wines are susceptible to downy mildew, the resistance needs to be introduced through breeding programs from P. viticola-resistant species found in the Vitaceae (Denzer et al. 1995; Staudt \& Kassemeyer 1995; Brown et al. 1999; Kortekamp \& Zyprian 2003; Cadle-Davidson 2008). As a consequence of several breeding efforts, knowledge about the genetic basis of the resistance to $P$. viticola has progressed in recent years (Marino et al. 2003; Merdinoglu et al. 2003; Welter et al. 2007; Bellin et al. 2009; Marguerit et al. 2009; Blasi et al. 2011).

Despite the advances in the characterisation of the resistance genes from the Vitaceae, knowledge about $P$. viticola genetics is scarce, and nothing is known about the nature of its avirulence genes. The identification of pathogen avirulence genes is a necessary step to understanding the biology of the interaction and has important implications in the breeding for disease resistance. In fact, identification of avirulence genes has proved useful for the discovery and functional profiling of disease resistance genes, allowing detection of functionally similar genes in different sources of resistance (Vleeshouwers et al. 2008). Furthermore, it has been proposed that studying the diversity and expression of putative avirulence genes in pathogen populations may provide valuable information when choosing the most efficient combination of resistance genes in order to achieve durable resistance (Michelmore 2003; Birch et al. 2008).

Most Oomycete avirulence proteins known to date belong to the RXLR family of effector proteins. They are small secreted proteins containing a signal peptide and an RXLR (Arg-X-LeuArg) motif (Kamoun 2006; Stassen \& van den Ackerveken 2011). Based on this information, P. viticola avirulence genes can be identified using a candidate gene strategy aimed at finding RXLR effectors by data mining of genomic resources. Unfortunately, public genomic resources of $P$. viticola are very limited. A recent search at EMBL/Genbank databases produced 83 P. viticola entries (ten ESTs and 73 core nucleotides), the majority corresponding to sequences of mitochondrial or ribosomal origin. Otherwise, cDNA-AFLP analysis of grapevine infected leaves produced $96 \mathrm{P}$. viticola sequences with an average size of 221 nucleotides (Polesani et al. 2008), and Solexa sequencing of cDNA derived from infected grapevine leaves attributed 251 short reads to $P$. viticola (Wu et al. 2010). The preliminary step of creation of $P$. viticola genomic resources is therefore required in order to identify putative avirulence genes in the interaction between grapevine and downy mildew.

Expression of Oomycete RXLR effectors is induced upon infection (Schornack et al. 2009, and references therein), so effector genes can be found in cDNA libraries from infected tissue at early stages of the interaction. Accordingly, identification of pathogen effectors by data mining of Expressed Sequence Tags (ESTs) derived from infected tissue has been reported for several pathosystems (Bittner-Eddy et al. 2003; Catanzariti et al. 2006; Cramer et al. 2006; Torto-Alalibo et al. 2007; Bowen et al. 2009; As-sadi et al. 2011; Cabral et al. 2011). However, this strategy involves sequencing a high number of cDNA clones since, despite the enrichment in putative effector genes, the pathogen biomass in the early stages of infection is still low compared to the plant biomass. An alternative to the use of infected tissues as the source of ESTs is using pathogen zoospores, which can be obtained in considerable amounts without difficulty, thus solving the problem of limited pathogen biomass. Plasmopara viticola zoospores are easily obtained by washing off sporangia from infected leaves and the first stages of pathogen development (growth of germinative tubes and vesicle formation) can be reproduced in vitro (Riemann et al. 2002). Interestingly, the expression of genes putatively involved in pathogenicity has been observed in zoospores from Oomycetes (Judelson \& Blanco 2005), opening the possibility of using this material to search for putative effectors.

In this paper we report on the development of ESTs resources for P. viticola. We obtained cDNA libraries from both $P$. viticola infected grapevine leaves and in vitro germinated zoospores, and evaluated the amount of pathogen sequences present in each library. To explore the suitability of in vitro germinated zoospores for the identification of pathogen effectors, we sequenced 1920 cDNA clones and identified 827 $P$. viticola nuclear ESTs. Sequence analysis revealed the presence of 54 ESTs from genes putatively involved in pathogenicity, together with other putative secreted proteins. Based on our results, in-depth analysis of the $P$. viticola transcriptome using next generation sequencing of cDNA from in vitro germinated zoospores should result in the identification of numerous candidate avirulence genes in the grapevine/downy mildew interaction.

\section{Materials and methods}

\section{Plant and pathogen materials}

Seedlings of Vitis vinifera cv. Muscat Ottonel were used in this study. Seedlings were grown on stone wool in a greenhouse at $22-19^{\circ} \mathrm{C}$ (day-night) and a photoperiod of $16 \mathrm{~h}$ of light.

Plasmopara viticola isolate SC was collected from $\mathrm{V}$. vinifera Chardonnay in the experimental field of INRA in Colmar (France) and maintained on detached leaves from seedlings of $\mathrm{V}$. vinifera cv. Muscat Ottonel.

\section{Sample preparation}

Plasmopara viticola-infected material for cDNA library construction was obtained by inoculating detached leaves as described in Peressotti et al. (2010). Briefly, leaves were surface-sterilized with bleach, followed by three washes in sterile water. Leaves were inoculated all through their surface with $10 \mu \mathrm{l}$-drops of a suspension of 50000 sporangia/ml, kept in Petri dishes on wet filter paper and incubated in a growing chamber at $21^{\circ} \mathrm{C}$ 
and a photoperiod of $16 / 8 \mathrm{~h}$ (light/dark, respectively). Fortyeight hours postinfection, four leaf discs were sampled and successful infection was confirmed by microscopic observation of pathogen structures using aniline-blue staining as described in Diez-Navajas et al. (2007). Leaves were then snap-frozen with liquid nitrogen and conserved at $-80^{\circ} \mathrm{C}$.

Plasmopara viticola-infected material for RT-PCR experiments was obtained by inoculating leaf discs as described in Peressotti et al. (2010). Leaf discs $1 \mathrm{~cm}$ in diameter were prepared from the fourth, fifth, and sixth leaf of Vitis vinifera seedlings, bulked, and placed abaxial surface up on humid filter paper on sealed Petri dishes. Discs were inoculated with $20 \mu \mathrm{l}$ of a suspension of 10000 sporangia/ml and incubated as described above. Samples for each time point consisted of a Petri dish containing ten leaf discs. At each time point, ten leaf discs were snap-frozen with liquid nitrogen and conserved at $-80^{\circ} \mathrm{C}$.

In vitro germinated $P$. viticola zoospores were obtained as described in Riemann et al. (2002). Detached leaves were infected as described above. Sporangia were washed off heavily infected leaves by gentle shaking in water in $50 \mathrm{ml} \mathrm{Fal-}$ con tubes. Leaves were removed and the suspension was left for $1 \mathrm{~h}$ at $21^{\circ} \mathrm{C}$ under light. After checking zoospore release with a microscope, $\mathrm{NaCl}$ was added to a final concentration of $10 \mathrm{mM}$ and the suspension was incubated for another hour under the same conditions. After checking zoospore germination with a microscope, zoospores were collected by centrifugation $15 \mathrm{~min}$ at $2200 \mathrm{~g}$ at $4{ }^{\circ} \mathrm{C}$. Zoospores were resuspended in $1 \mathrm{ml}$ of sterile distilled water and transferred to 1.5-ml Eppendorf tubes. Suspensions were centrifuged for 2 min at maximum speed using a microcentrifuge and the supernatant was removed by aspiration. Tubes were snapfrozen with liquid nitrogen and conserved at $-80^{\circ} \mathrm{C}$.

\section{RNA extraction}

Total RNA was extracted from infected leaves as previously described (Zeng \& Yang 2002). RNA extractions from Plasmopara viticola in vitro germinated zoospores were performed using the RNeasy Plant Mini Kit (Qiagen) following the manufacturer's instructions. Removal of residual genomic DNA was achieved by DNase treatment of RNAs using the Ambion Turbo-DNAfree kit. RNAs were quantified using a Nanodrop ND-1000 spectrophotometer (Thermo Scientific).

\section{Library construction}

Both cDNA libraries were obtained in phagemid vector $\lambda$ TriplEx2 using Clontech's SMART ${ }^{\text {тM }}$ CDNA Library Construction Kit. cDNAs were synthesized from $1 \mu \mathrm{g}$ of total RNA by LDPCR using 20 cycles of amplification. cDNAs were then digested with SfiI, size-fractionated, and ligated to vector $\lambda$ TriplEx2 following the manufacturer's instructions with all appropriate controls. Packaging reactions were performed using MaxPlax ${ }^{\top M}$ Lambda Packaging Extracts from Epicentre Biotechnologies. Titering of the unamplified library, library amplification, and titering of the amplified library were performed following Clontech's SMART ${ }^{\mathrm{TM}}$ CDNA Library Construction Kit instructions using Escherichia coli strain XL1-Blue. The library derived from germinated zoospores showed an unamplified titre of $6 \times 10 \mathrm{e} 6 \mathrm{pfu} \mathrm{ml} \mathrm{m}^{-1}(7 \times 10 \mathrm{e} 8$ amplified). The library derived from infected tissue showed an unamplified titre of $3 \times 10 \mathrm{e} 7(2 \times 10 \mathrm{e} 9$ amplified $) \mathrm{pfu} \mathrm{m}^{-1}$. Library insert size was evaluated on 24 individual phage plaques by PCR with M13 universal primers: isolated phage plaques were placed in $200 \mu \mathrm{l}$ of $1 \times$ lambda dilution buffer, vortexed and then $1 \mu \mathrm{l}$ was used in a $20 \mu \mathrm{l}$ PCR reaction. Amplified libraries were conserved in DMSO (Dimethyl sulfoxide) at $-80^{\circ} \mathrm{C}$. Bulk conversion of phagemid $\lambda$ TriplEx2 clones to plasmid pTriplEx2 clones was performed following the kit instructions using $E$. coli strain BM25.8. This required performing preliminary tests aimed at finding the amount of amplified lysate producing a satisfactory density for colony picking. Ten thousand colonies were picked, grown on 384-well plates in freezing media supplemented with carbenicillin, and stored at $-80^{\circ} \mathrm{C}$.

\section{Sequence analysis}

Sequencing of 1920 cDNA clones on plasmid pTriplEx2 was outsourced to GATC Biotech, Konstanz, Germany. cDNA sequences were assembled using the GAP4 module of Staden package 1.6.0 (http://sourceforge.net/projects/staden) (Staden 1996). Library redundancy was calculated as described in Cramer et al. (2006). Identification of grapevine sequences was performed by nucleotidic Blast against the $12 \mathrm{X}$ version of the grapevine genome sequence (http:/www.genoscope.cns.fr/cgi-bin/blast_ server/projet_ML/blast.pl) (Jaillon et al. 2007); sequences showing identity $\geq 95 \%$ to the grapevine genome sequence were considered as derived from grapevine. Sequences belonging to Plasmopara viticola ribosomal RNAs or mitochondrion, as well as sequences from bacterial origin, were selected after nucleotidic Megablast against the $\mathrm{nr} / \mathrm{nt}$ database at the National Center for Biotechnology Information (NCBI) (http://blast.ncbi.nlm. nih.gov/Blast.cgi) (Altschul et al. 1990). Sequences were translated using EMBOSS Transeq at the European Bioinformatics Institute (EBI) (http://www.ebi.ac.uk/Tools/emboss/transeq/). Functional identification was done by BlastX analysis against the nonredundant (nr) protein database at NCBI and against the Phytophthora infestans proteome at the Broad Institute (http://www.broadinstitute.org/annotation/genome/phytophthora_ infestans/Blast.html) (Haas et al. 2009). Although the genome sequence from other Oomycetes is available, we limited our search to just one of them for the sake of clanity, since preliminary analysis using other Oomycete genome sequences revealed similar results. Motif identification and Gene Ontology (GO) classification were performed using InterProScan at EBI (http:// www.ebi.ac.uk/Tools/pfa/iprscan/) (Hunter et al. 2009). Putative secreted proteins were identified using SignalP at CBS (http:// www.cbs.dtu.dk/services/SignalP/) (Emanuelsson et al. 2007).

The 563 putative nuclear $P$. viticola unigenes had an average size of $580 \mathrm{nt}, 530 \mathrm{nt}$ when considering only the 454 singletons. Sequences from unigenes possessing at least two ESTs as well as from other singletons cited in the manuscript have been submitted to the EMBL database with accession numbers HE582023 to HE582206.

\section{Semiquantitative RT-PCR}

First strand synthesis was performed using RevertAid First Strand cDNA Synthesis kit following the manufacturer instructions (Fermentas). PCR amplification was done using 
GoTaq Taq Polymerase (Promega). PCRs for Vitis vinifera actin consisted of 25 cycles of $20 \mathrm{~s}$ at $94^{\circ} \mathrm{C}, 20 \mathrm{~s}$ at $60{ }^{\circ} \mathrm{C}$, and $45 \mathrm{~s}$ at $72{ }^{\circ} \mathrm{C}$, followed by a final extension of $10 \mathrm{~min}$ at $72{ }^{\circ} \mathrm{C}$. PCRs for Plasmopara viticola genes consisted of 30 cycles of the same programme. Primer sequences for grapevine and $P$. viticola actins have been previously reported (Schmidlin et al. 2008). Primer sequences for other $P$. viticola genes are available upon request. PCR products were resolved in $1 \%(\mathrm{w} / \mathrm{v})$ agarose gels in TAE (40 mM Tris-acetate, 1 mM EDTA, pH 8) and visualised by ethidium bromide staining.

\section{Results}

cDNA library construction and sequencing

Two cDNA libraries were obtained in the phagemid vector $\lambda$ TriplEx2, one from in vitro germinated Plasmopara viticola zoospores (Fig 1A and B) and a second from infected nonsporulating grapevine leaves (Fig 1C). Average insert sizes were $750 \mathrm{nt}$ for the germinated zoospores library and $900 \mathrm{nt}$ for the library derived from infected tissue. The percentage of empty clones was $5 \%$ for both libraries.
Inserts from 45 phage plaques from each library were 5 'end-sequenced using a $\lambda$ TriplEx2-specific primer. The availability of the grapevine genome sequence as well as the sequences from different Oomycetes allowed us to attribute an origin to the sequences. Thus, sequence analysis of the zoospore library revealed $6.6 \%$ (3/45) of grapevine sequences and $62.2 \%(28 / 45)$ of sequences that could be attributed to $P$. viticola, while the remaining $31 \%(14 / 45)$ did not show significant hits on the selected databases. The library from infected tissue showed $82.2 \%$ (37/45) of grapevine sequences and $17.7 \%(8 / 45)$ of putative $P$. viticola sequences. Despite the high density of the infection in the tissues used for the interaction library (Fig 1C) the percentage of $P$. viticola sequences was too low, so we concentrated our efforts on the germinated zoospore library.

An aliquot of the germinated zoospore library was used to convert the $\lambda$ TriplEx2 phage clones into pTriplEx2 plasmid clones. Ten thousand colonies were picked and stored in 384-well plates. One thousand nine hundred twenty clones were 5 'end-sequenced using a pTriplEx2-specific primer, producing 1543 useful sequences. Sequences were assembled using the GAP4 module from Staden and analysed as described in the flowchart in Fig 2.
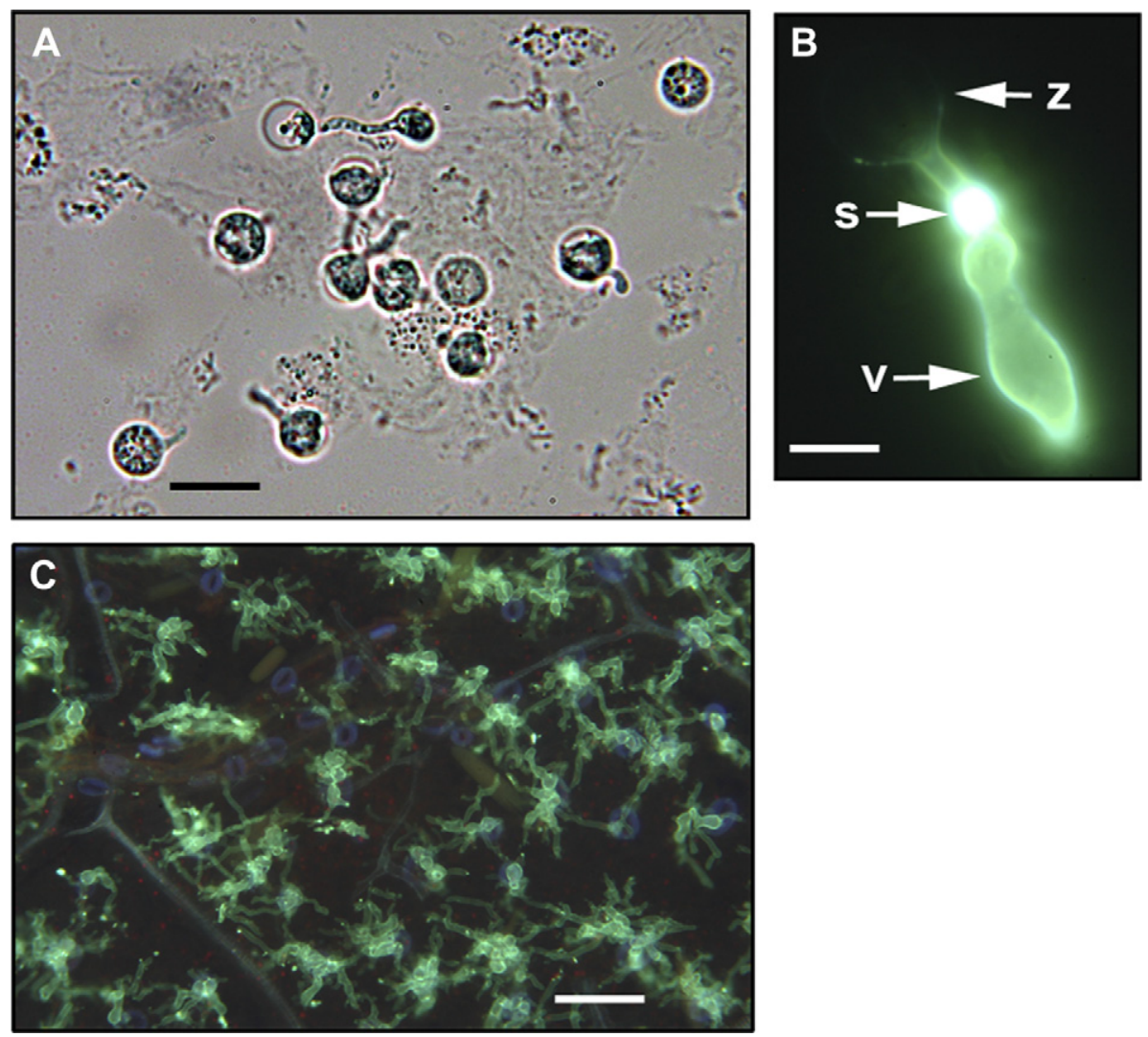

Fig 1 - Tissues used for the construction of P. viticola cDNA libraries.

(A) In vitro germinated zoospores from P. viticola. Zoospore cytoplasm appears in grey. Picture taken under a bright light microscope. Bar $=10 \mu \mathrm{m}$. (B) Detail of a germinated zoospore stained with aniline blue observed under UV light. P. viticola structures appear green fluorescent. z: zoospore, s: septum, v: vesicle. Picture taken under an epifluorescence microscope. Bar $=5 \mu \mathrm{m}$. (C) Aniline-blue stained grapevine leaves infected with P. viticola. Picture taken at $48 \mathrm{hpi}$ using an epifluorescence microscope. P. viticola structures appear green fluorescent. Bar $=\mathbf{5 0} \mu \mathrm{m}$. (For interpretation of the references to colour in this figure legend, the reader is referred to the web version of this article.) 


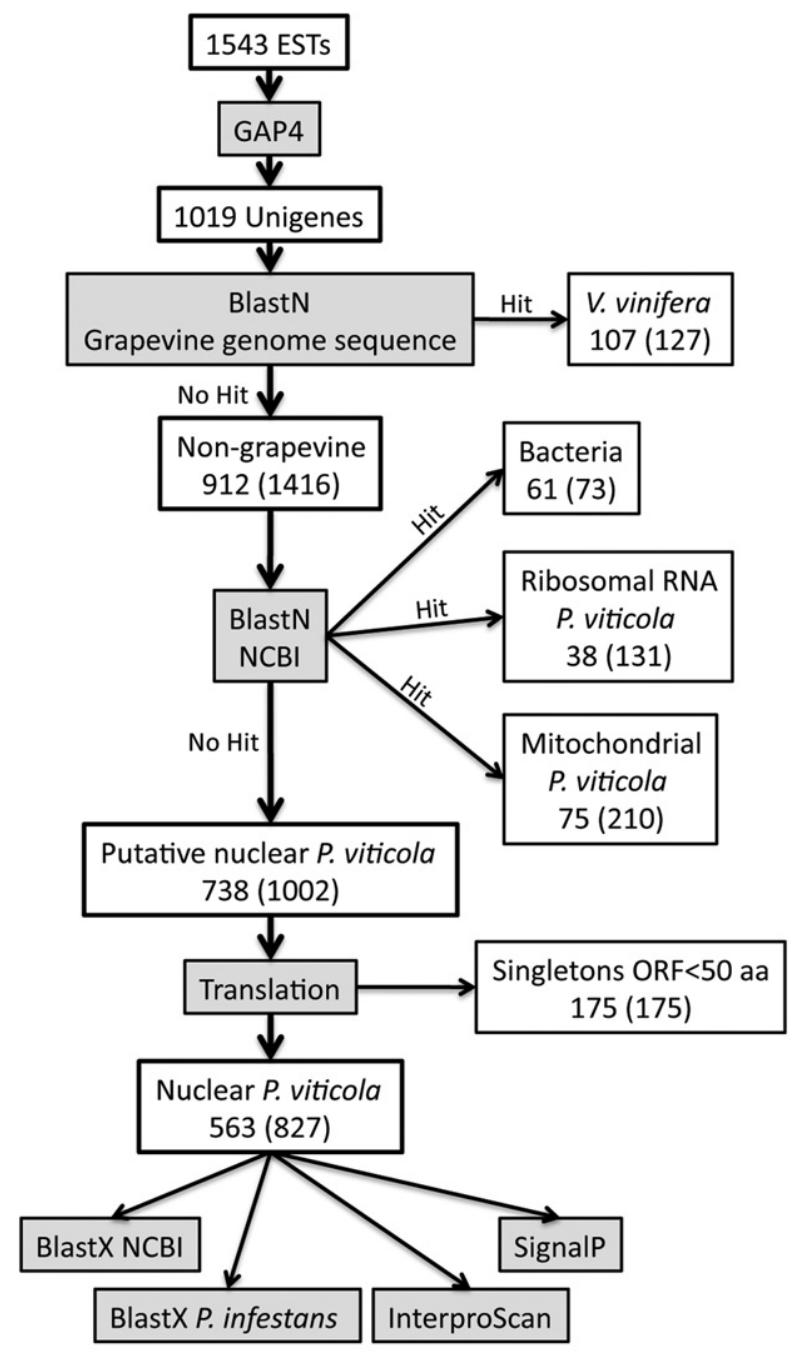

Fig 2 - Flowchart of $P$. viticola cDNA sequence analysis. Grey shaded boxes show analysis procedures. White boxes show sets of unigenes, with EST numbers shown between brackets. ESTs were assembled with GAP4. Nucleotidic Blast against the grapevine genome sequence allowed removing of grapevine sequences. Nucleotidic Blast against the NCBI $\mathrm{nr}$ database allowed identifying sequences from $P$. viticola mitochondrion and ribosomal RNA, as well as other contaminant sequences. Putative $P$. viticola nuclear sequences were translated in three ORFs and singletons producing peptides smaller than $\mathbf{5 0}$ aminoacids were discarded. The remaining sequences were subjected to the analysis shown at the bottom of the flowchart.

\section{Functional classification of sequences}

Results of sequence analysis are summarized in Table 1 . Sequence analysis identified 563 putative nuclear Plasmopara viticola unigenes corresponding to 827 ESTs, with 109 unigenes possessing at least two ESTs and 454 singletons, thus giving a level of redundancy of $32 \%$. Blast hits to the NCBI nr protein database and to the Phytophthora infestans proteome were obtained for $58 \%$ and $82 \%$ of unigenes respectively, which
Table 1 - Distribution of putative $P$. viticola nuclear cDNA sequences from the in vitro germinated zoospores library.

\begin{tabular}{|c|c|c|}
\hline & Unigenes (\%) & ESTs (\%) \\
\hline Total & $563(100)$ & $827(100)$ \\
\hline 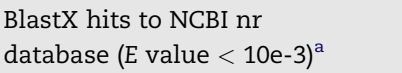 & $325(58)$ & $535(65)$ \\
\hline $\begin{array}{l}\text { BlastX hits to } P \text {. infestans } \\
\text { proteome }(E \text { value }<10 \mathrm{e}-3)^{\mathrm{a}}\end{array}$ & $465(82)$ & $698(84)$ \\
\hline $\begin{array}{l}\text { BlastN hits to } P \text {. infestans } \\
\text { transcriptome }(E \text { value }<10 \mathrm{e}-3)^{\mathrm{b}}\end{array}$ & $360(64)$ & $535(65)$ \\
\hline Hits InterPro ${ }^{c}$ & $226(40)$ & $395(48)$ \\
\hline Hits GO molecular function ${ }^{c}$ & $159(28)$ & $278(34)$ \\
\hline Hits GO biological process ${ }^{c}$ & $119(21)$ & $163(20)$ \\
\hline Hits GO cellular compartment ${ }^{\mathrm{c}}$ & $51(9)$ & $76(9)$ \\
\hline Predicted secreted (SignalP) & $36(6)$ & $117(14)$ \\
\hline $\begin{array}{l}\text { Putative secreted (SignalP }+ \\
\text { similarity } P . \text { infestans }{ }^{d} \text { ) }\end{array}$ & $81(14)$ & $179(22)$ \\
\hline \multicolumn{3}{|c|}{$\begin{array}{l}\text { a Sequences showing similarity at the protein level. } \\
\text { b Sequences showing similarity at the nucleotide level. } \\
\text { c Obtained by submitting the protein sequence to InterProScan. } \\
\text { d Proteins highly similar ( } E \text { value }<1 \text { e-30) to P. infestans secreted } \\
\text { proteins. }\end{array}$} \\
\hline
\end{tabular}

is in accordance with our sequences being from Oomycete origin. Manual inspection of the alignments with the P. infestans proteome revealed that most ESTs possessed the cDNA $3^{\prime}$-end, while a considerable number were missing the $5^{\prime}$-end.

We performed functional classification of ESTs based on the GO annotation obtained with InterProScan. Biological processes could be tentatively assigned to 119 unigenes. Organisation of the different biological processes in categories is shown in Table 2. Genes involved in metabolism, with particular emphasis on cell wall metabolism, and other housekeeping processes accounted for almost half of the hits. Other well represented processes were protein degradation, protein phosphorylation/signalling, and transport. A total of 226 unigenes showed hits to protein signatures from the InterPro databases. Highly represented protein signatures are listed in Table 3.

SignalP analysis identified 36 unigenes (117 ESTs) possessing a signal peptide and thus encoding putatively secreted proteins. Since a number of our ESTs were missing the $5^{\prime}$ end and thus precluded us from checking for the presence of a signal peptide, we searched for sequences showing high similarity ( $E$ value $<10 \mathrm{e}-30$ ) to $P$. infestans proteins predicted to be secreted. Altogether we found 81 unigenes (179 ESTs) coding for putatively secreted proteins, out of which 26 unigenes (54 ESTs) were coding for proteins with functions and/or motifs putatively involved in pathogenesis (Table 4).

\section{Expression analysis of selected genes}

To verify that the putative effectors found in germinated zoospores were actually being expressed in infected tissue, we selected five genes encoding putative secreted full-length candidate effectors and studied their expression in different developmental stages of Plasmopara viticola by semiquantitative RT-PCR. The selected genes coded for a protein with Kazal-like protease inhibitor fold, an INL11B-like elicitin, an 
Table 2 - Functional classification of P. viticola cDNA sequences from in vitro germinated zoospores based on annotation with InterProScan.

\begin{tabular}{|c|c|c|c|}
\hline Process & Unigenes (ESTs) & GO biological process & Unigenes (ESTs) \\
\hline \multirow[t]{13}{*}{ Metabolism } & \multirow[t]{13}{*}{$35(46)$} & Carbohydrate metabolic process (GO:0005975) & $6(6)$ \\
\hline & & Metabolic process (GO:0008152) & $4(5)$ \\
\hline & & Oxidation reduction (GO:0055114) & $7(7)$ \\
\hline & & Aminoacid metabolism & $3(4)$ \\
\hline & & ATP biosynthetic process (GO:0006754) & $3(5)$ \\
\hline & & PI metabolic process (GO:0046488) & $3(3)$ \\
\hline & & Tricarboxylic acid cycle (GO:0006099) & $2(2)$ \\
\hline & & Protein metabolic process (GO:0019538) & $2(3)$ \\
\hline & & Lipid metabolic process (GO:0006629) & $1(2)$ \\
\hline & & Inositol biosynthetic process (GO:0006021) & $1(5)$ \\
\hline & & Glyoxylate cycle (GO:0006097) & $1(2)$ \\
\hline & & Cyclic nucleotide biosynthetic process (GO:0009190) & $1(1)$ \\
\hline & & Ceramide metabolic process (GO:0006672) & $1(1)$ \\
\hline \multirow[t]{3}{*}{ Cell wall metabolism } & \multirow[t]{3}{*}{$8(15)$} & Cell wall catabolic process (GO:0016998) & $3(6)$ \\
\hline & & Cell wall modification (GO:0042545) & $3(3)$ \\
\hline & & Cellulose catabolic process (GO:0030245) & $2(6)$ \\
\hline \multirow[t]{8}{*}{ Transport } & \multirow[t]{8}{*}{$18(20)$} & Transport (GO:0006810) & $2(3)$ \\
\hline & & Transmembrane transport (GO:0055085) & $5(5)$ \\
\hline & & Anion transport (GO:0006820) & $1(1)$ \\
\hline & & Cation transport (GO:0006812) & $1(2)$ \\
\hline & & Potassium ion transport (GO:0006813) & $2(2)$ \\
\hline & & Metal ion transport (GO:0030001) & $2(2)$ \\
\hline & & Intracellular protein transport (GO:0006886) & $3(3)$ \\
\hline & & Vesicle-mediated transport (GO:0016192) & $2(2)$ \\
\hline \multirow[t]{5}{*}{ Signalling } & \multirow[t]{5}{*}{$12(15)$} & Cell communication (GO:0007154) & $5(8)$ \\
\hline & & Small GTPase mediated signal transduction (GO:0007264) & $3(3)$ \\
\hline & & G-protein coupled receptor signalling pathway (GO:0007186) & $2(2)$ \\
\hline & & Signal transduction (GO:0007165) & $1(1)$ \\
\hline & & Two-component signal transduction system (GO:0000160) & $1(1)$ \\
\hline \multirow[t]{3}{*}{ Protein phosphorylation } & \multirow[t]{3}{*}{$14(17)$} & Protein amino acid phosphorylation (GO:0006468) & $12(13)$ \\
\hline & & Protein amino acid dephosphorylation (GO:0006470) & $1(1)$ \\
\hline & & Regulation of protein amino acid phosphorylation (GO:0001932) & $1(3)$ \\
\hline \multirow[t]{2}{*}{ Protein degradation } & \multirow[t]{2}{*}{$13(22)$} & Proteolysis (GO:0006508) & $9(16)$ \\
\hline & & Protein ubiquitination (GO:0016567) & $4(6)$ \\
\hline \multirow[t]{6}{*}{ Others } & \multirow[t]{6}{*}{$19(28)$} & Translation (GO:0006412) & $9(11)$ \\
\hline & & Transcription (GO:0006350) & $2(3)$ \\
\hline & & Protein folding (GO:0006457) & $2(2)$ \\
\hline & & Microtubule-based movement (GO:0007018) & $3(4)$ \\
\hline & & Pathogenesis (GO:0009405) & $2(7)$ \\
\hline & & DNA repair (GO:0006281) & $1(1)$ \\
\hline
\end{tabular}

Table 3 - Protein signatures highly represented in the cDNA library from $P$. viticola in vitro germinated zoospores.

\begin{tabular}{lcc} 
Function/Motif & InterPro & Unigenes (ESTs) \\
\hline Hexapeptide transferase & IPR018357 & $12(49)$ \\
MCO/Laccase & IPR008972 & $1(26)$ \\
Mannose-binding lectin & IPR001229 & $5(15)$ \\
EGF-like region & IPR013032 & $3(10)$ \\
Tubby & IPR000007 & $2(9)$ \\
Phox homologous domain & IPR001683 & $4(7)$ \\
ABC-2 type transporter & IPR013525 & $3(7)$ \\
Adventurous gliding, peptidase & IPR009003 & $1(7)$ \\
Cellulose synthase & IPR005150 & $4(5)$ \\
Myo-inositol 1-phosphate synthase & IPR002587 & $1(5)$ \\
Total & & $36(140)$ \\
\hline
\end{tabular}

RXLR protein, a mannose-binding lectin, and an acidic chitinase. Expression was studied on $P$. viticola sporangia, in vitro germinated zoospores, and in infected grapevine leaves at different times postinoculation $(6,24,48,72$, and $96 \mathrm{~h}$ postinoculation (hpi), the last corresponding to sporulating leaves). Expression of $P$. viticola actin was used to monitor the infection process and evaluate pathogen biomass, while Vitis vinifera actin was used to standardise results among the samples corresponding to infected leaves.

Results are shown in Fig 3. The steady increase in expression of $P$. viticola actin in the infection samples confirmed successful pathogen colonisation over time and showed the semiquantitative nature of the RT-PCR. Expression of elicitin, Kazal-like inhibitor, and RXLR genes could be detected in infected tissues, whilst chitinase and mannose-binding lectin were barely detectable. The genes coding for elicitin and 
Table 4 - P. viticola ESTs corresponding to putative secreted hydrolytic enzymes and effectors.

\begin{tabular}{|c|c|c|}
\hline Function/Motif & Unigenes (ESTs) & BlastX $P$. infestans (E value) ${ }^{a}$ \\
\hline \multirow[t]{2}{*}{ Glucanase inhibitor protein } & $4(9)$ & PITG_13655 (4e-82; 1e-15) \\
\hline & & PITG_13671 (1e-26; 9e-17) \\
\hline RXLR-dEER protein & $2(9)$ & $\mathrm{NS}^{\mathrm{b}}$ \\
\hline Elicitin-like & $2(7)$ & PITG_10772 (6e-41); PITG_19604 (3e-42) \\
\hline \multirow[t]{2}{*}{ Endo-1,3-ß-glucanase } & $3(5)$ & PITG_03511 (1e-106); PITG_03511 (6e-72) \\
\hline & & PITG_15980 (1e-108) \\
\hline Endo-1,4-ß-glucanase & $2(6)$ & PITG_18336 (2e-41; 2e-22) \\
\hline Secretory protein OPEL & $2(4)$ & PITG_00891 (5e-39); PITG_05156 (2e-37) \\
\hline \multirow[t]{2}{*}{ Pectinesterase } & $3(3)$ & PITG_18907 (3e-97; 1e-52) \\
\hline & & PITG_08912 (6e-35) \\
\hline Transglutaminase elicitor & $2(3)$ & PITG_16956 (1e-87); PITG_16958 (1e-39) \\
\hline Acidic chitinase & $1(3)$ & PITG_17947 (1e-108) \\
\hline Cathepsin-like cysteine protease & $2(2)$ & PITG_13074 (6e-99); PITG_02423 (1e-161) \\
\hline Cystatin-like cysteine protease inhibitor & $2(2)$ & PITG_09169 (2e-10); PITG_00058 (2e-05) \\
\hline Kazal-like protease inhibitor & $1(1)$ & PITG_09840 (6e-23) \\
\hline Total & $26(54)$ & \\
\hline
\end{tabular}

a Best hit with the P. infestans proteome for each of the unigenes.

b NS: no significant hits found at $E$ value $\leq 0.01$.

Kazal-like inhibitor were detected as soon as 0 hpi (samples taken just after inoculation) and their expression rose with the increase in pathogen biomass. The gene coding for an RXLR protein was detected as early as $6 \mathrm{hpi}$, its expression peaked at $24-48 \mathrm{hpi}$ and decreased at later time points. Elicitin, Kazal-like inhibitor, and RXLR were detected at earlier time points than actin, suggesting an early induction of expression of secreted proteins. Expression of all genes could be detected in $P$. viticola sporangia as well as in germinated zoospores.

\section{Discussion}

In this paper we described the development and analysis of ESTs from in vitro germinated zoospores of Plasmopara viticola, the causal agent of grapevine downy mildew. We report 827 ESTs clustered in 563 unigenes. Sequence analysis revealed an important number of housekeeping genes, involved in processes such as metabolism, protein degradation, phosphorylation, signalling, and transport (Table 2). Despite the low redundancy of the library, some protein functions and motifs were represented by several ESTs, implying a potential role for these functions in the early stages of $P$. viticola development.

The library was rich in proteins containing the hexapeptide repeat motif (IPR018357; 49 ESTs corresponding to 12 unigenes) found in different transferase protein families, such as galactoside acetyltransferase-like proteins, the gamma-class of carbonic anhydrases, and tetrahydrodipicolinate- $\mathrm{N}$-succinlytransferases (Vaara 1992; Jenkins \& Pickersgill 2001). However, the P. viticola sequences lack particular transferase motifs, thus hindering us from making any hypothesis about the putative role these proteins may play in the infection process.

The most represented unigene (26 ESTs) encodes a protein showing cuproredoxin fold motifs (IPR008972) and thus belonging to the Multicopper Oxidase Blue Protein (MCBPs) family (Nakamura \& Go 2005). The most similar Phytophthora infestans protein (PITG 17323, $60 \%$ aa identity, E value $=1 \mathrm{e}$ -
166) possesses three cuproredoxin domains and is predicted to be secreted. The presence of three cuproredoxin domains in the predicted protein places it into the Multicopper Oxidases (MCOs), which are involved in the oxidization of a variety of aromatic substrates and include, among others, laccase and ascorbate oxidase (Nakamura \& Go 2005). While ascorbate oxidase is found only in plants, laccases are found in a variety of organisms such as bacteria, fungus, plants, and insects. Among these, fungal laccases are the best characterised, mainly due to their role in lignin degradation. Other than their role in lignin catabolism, fungal laccases have been shown to be involved in developmental processes, pigment formation, and pathogenesis (Hoegger et al. 2006). Nothing is known about laccases from Oomycetes. Based on the results of Blast analysis, the $P$. viticola sequence shows the best similarity to fungal laccases. Interestingly, a laccase-like protein from the fungal pathogen Botrytis cinerea has been shown to oxidize pterostilbene and resveratrol (Pezet et al. 1991), two grapevine phytoalexins that are synthesized in response to pathogen attack and have been suggested to play a role against grapevine pathogens, including downy mildew (Pezet et al. 2004). Therefore, although it remains possible that this protein is involved in the developmental processes associated with zoospore germination, it is tempting to speculate on its possible function in stilbene detoxification and thus its contribution to pathogenesis.

We found 15 ESTs corresponding to five unigenes coding for Jacalin-like mannose-binding lectins (IPR001229) possessing a signal peptide. Lectins are proteins found in organisms from all kingdoms characterised for binding specific carbohydrates reversibly. In animals, intracellular lectins are involved in protein quality control (Lederkremer 2009), while cell surface lectins play a role in adhesion, cell-to-cell interactions, and innate immunity (Fujita 2002). Plant lectins, which are mostly extracellular, are believed to play a role in interactions with other organisms, either in defence or in recognition, and Jacalin-related lectins have been described to play a role as storage proteins and/or in defence (van Damme et al. 2004; 


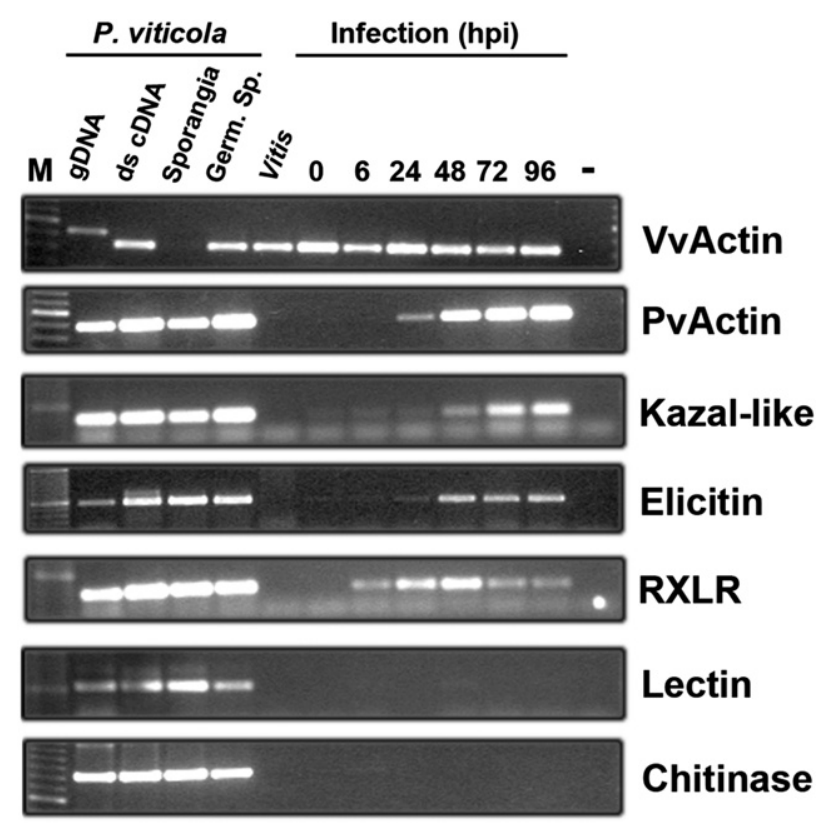

Fig 3 - Expression analysis of selected P. viticola secreted genes upon infection.

The first two lanes correspond to PCRs on P. viticola gDNA and double stranded cDNA from in vitro germinated zoospores, respectively. The remaining lanes show RT-PCRs on P. viticola sporangia (Sporangia), in vitro germinated zoospores (Germ. Sp.), V. vinifera (Vitis), and P. viticola-inoculated $V$. vinifera from 0 to $96 \mathrm{~h}$ postinfection (Infection (hpi)). (-): PCR negative control. M: molecular weight marker. VvACT: V. vinifera actin. PvACT: P. viticola actin. Note V. vinifera actin expression is also detected in P. viticola zoospores due to the presence of contaminating plant material in the zoospores preparation. The different size of VVACT in the first lane is caused by the presence of an intron in the actin sequence. Results are representative of two independent experiments. Accession numbers for these genes are: HE582205 (Kazal-like protease inhibitor), HE582038 (INL11Blike elicitin), HE582030 (RXLR-like protein), HE582037 (mannose-binding lectin), and HE582051 (acidic chitinase).

de Hoff et al. 2009). Little is known about lectins from plant pathogens. RSA (Rhizoctonia solani agglutinin) from the plant pathogenic fungus $R$. solani is a lectin abundant in the fungal survival structures that has been reported to be toxic against the cotton leaf worm (Hamshou et al. 2010). Although we cannot rule out the possibility that $P$. viticola lectins play a role in host recognition and cellular adhesion, the low level of expression found for one of the $P$. viticola Jacalin-like lectins in infected tissue (Fig 3) suggests that, rather than being involved in the interaction with the plant, these proteins might function as storage proteins in zoospores, with putative roles in defence against pathogens in the early stages of $P$. viticola development. In this context, it is worth noting that the P. viticola lectin and chitinase genes show similar expression patterns (Fig 3), and chitinases are known to function in defence against fungal pathogens.
The library contained several genes related to inositol metabolism and inositol-mediated membrane trafficking. We found five ESTs from Myo-inositol 1-phosphate synthase (IPR002587), which catalyses the rate-limiting first step of myo-inositol biosynthesis from glucose-6-phosphate (Michell 2008). Being a precursor of phosphatidylinositol (PI), the role of myo-inositol biosynthesis in the early stages of $P$. viticola development could be contributing the PI required for the membrane synthesis associated to pathogen growth. A second possibility is that PI is used as a precursor for the synthesis of glycosylphosphatidylinositol (GPI) anchors (Michell 2008); interestingly, we found six ESTs from an elicitin-like gene (IPR002200) that is conserved among Oomycetes and predicted to be GPI-anchored. Finally, a more appealing possibility is that PI is used as the precursor of phosphorylated signaltransduction molecules playing an important role in membrane trafficking pathways (Odorizzi et al. 2000). A detailed analysis of the ESTs revealed the presence of several genes involved in the metabolism of PI phosphorylated derivatives: PI4-kinase (IPR000403, two ESTs), catalysing the synthesis of PI 4-phosphate from PI; PI 4-phosphate 5 kinase (IPR002498, one EST) catalysing the synthesis of PI 4,5-bisphosphate from PI 4-phosphate; PI 3,4-bisphosphate phosphatase (two ESTs), involved in the turnover of PI 3,4-bisphosphate. In accordance with a possible role of PI in membrane trafficking, we found proteins containing inositol-binding domains, like the Phox and FYVE domains (seven ESTs and two ESTs respectively), eight ESTs from Tubby, a protein known to bind inositol (Santagata et al. 2001), and several components of membrane trafficking, like clathrin adaptor protein (three ESTs), SNARE protein, Rab GTPase, and Sec-like vesicular fusion protein (Odorizzi et al. 2000). It is noteworthy that PI 4-phosphate has been involved in the regulation of secretion (Hama et al. 1999).

Plant pathogenic fungi and Oomycetes secrete an array of hydrolytic enzymes and effectors to manipulate host structure and metabolism and thus accomplish successful infection (Kamoun 2006; de Jonge et al. 2011). Identifying these secreted proteins and knowing their mode of function is important to understand the establishment of compatibility between host and pathogen. Also, since most avirulence genes corresponding to resistance genes against fungal or Oomycete pathogens are secreted proteins (Stassen \& van den Ackerveken 2011), identifying the pathogen's secretome opens the way to discovering the cognate avirulence genes using a candidate gene approach. The $P$. viticola cDNA library contained 117 ESTs corresponding to 36 unigenes that encoded proteins predicted to contain a signal peptide and thus being secreted. When considering the proteins whose best Blast hit on $P$. infestans ( $E$ value $<10 \mathrm{e}-30$ ) were secreted, the number of putatively secreted proteins rose to 81 (179 ESTs). These numbers stand for $6 \%$ and $14 \%$ of the $P$. viticola unigenes respectively (Table 1). Analysis of Hyaloperonospora arabidopsidis ESTs from an infection cDNA library revealed that $9 \%$ of the pathogen unigenes were predicted to be secreted (Cabral et al. 2011), and Phytophthora sojae ESTs derived from cDNA libraries corresponding to different pathogen developmental stages contained $8 \%$ of secreted unigenes (Torto-Alalibo et al. 2007). Thus, the percentage of secreted proteins observed for the $P$. viticola germinated zoospores library appears slightly higher than for other Oomycetes, although these differences could be associated with the type of biological material used for the different libraries. 
Importantly, among these putatively secreted proteins we found 26 unigenes (54 ESTs) corresponding to hydrolytic enzymes, protein inhibitors, elicitor-like proteins, and members of the RXLR family of effectors (Table 4). RT-PCR experiments at different stages of the $P$. viticola infection cycle showed that some of these genes were actually expressed upon infection (Fig 3). Expression of RXLR proteins and apoplastic effectors is known to be upregulated upon infection (Haas et al. 2009). The kinetics of expression observed for the P. viticola RXLR gene fits with current knowledge about RXLR effectors, which are strongly expressed in the early stages of the interaction and also in germinated zoospores (Dong et al. 2009; Haas et al. 2009). Expression of the Kazal-like proteinase inhibitor increased with pathogen biomass, with a pattern similar to that reported for the $P$. infestans Kazal-like protease inhibitor epi1 (Tian et al. 2004). Concerning the INL11B-like elicitin, ESTs from the homologous $P$. infestans inl11b and $P$. sojae sol11 $b$ have been found in cDNA libraries derived from spores and infection, respectively (Jiang et al. 2006), and the P. viticola INL11B-like gene expression pattern was comparable to those of $P$. infestans elicitins inf1 and inf2b (Huitema et al. 2005). Altogether, these results confirm the usefulness of in vitro germinated zoospores for the identification of genes involved in the interaction between grapevine and P. viticola.

\section{Conclusion}

Here we report the construction of a cDNA library from in vitro germinated zoospores from the Oomycete Plasmopara viticola and show the suitability of this material for the analysis of the $P$. viticola secretome and subsequent identification of genes involved in pathogenicity. High-throughput sequencing of cDNA from in vitro germinated zoospores should allow in-depth analysis of the P. viticola secretome, allowing not only a better understanding of the interaction but also the identification of avirulence genes corresponding to the different grapevine resistance genes. The identification of effectors from $P$. viticola and the characterisation of some of them as avirulence genes will allow us to perform functional profiling of resistance sources as well as to choose the best combinations of genes in a strategy of pyramiding, thus improving the efficiency of the breeding programs for resistance to grapevine downy mildew.

\section{Acknowledgements}

We thank Maria Santos-Rosa for help in sample preparation, Delphine Jublot for technical assistance in colony picking and Jean-François Chich for critical reading of the manuscript. Research funded in part by INRA's Department of Plant Genetics and Breeding (DGAP).

\section{R E F E R E N C E S}

Altschul S, Gish W, Miller W, Myers E, Lipman D, 1990. Basic local alignment search tool. Journal of Molecular Biology 215: 403-410.
As-sadi F, Carrere S, Gascuel Q, Hourlier T, Rengel D, Le Paslier M-C, Bordat A, Boniface M-C, Brunel D, Gouzy J, Godiard L, Vincourt P, 2011. Transcriptomic analysis of the interaction between Helianthus annuus and its obligate parasite Plasmopara halstedii shows single nucleotide polymorphisms in CRN sequences. BMC Genomics 12: 498.

Bellin D, Peressotti E, Merdinoglu D, Wiedemann-Merdinoglu S, Adam-Blondon A-F, Cipriani G, Morgante M, Testolin R, Di Gaspero G, 2009. Resistance to Plasmopara viticola in grapevine 'Bianca' is controlled by a major dominant gene causing localised necrosis at the infection site. Theoretical and Applied Genetics 120: 163-176.

Birch P, Boevink P, Gilroy E, Hein I, Pritchard L, Whisson S, 2008. Oomycete RXLR effectors: delivery, functional redundancy and durable disease resistance. Current Opinion in Plant Biology 11: 373-379.

Bisson L, Waterhouse A, Ebeler S, Walker M, Lapsley J, 2002. The present and future of the international wine industry. Nature 418: 696-699.

Bittner-Eddy PD, Allen RL, Rehmany AP, Birch P, Beynon JL, 2003. Use of suppression subtractive hybridization to identify downy mildew genes expressed during infection of Arabidopsis thaliana. Molecular Plant Pathology 4: 501-507.

Blasi P, Blanc S, Wiedemann-Merdinoglu S, Prado E, Ruhl E, Mestre P, Merdinoglu D, 2011. Construction of a reference linkage map of Vitis amurensis and genetic mapping of Rpv8, a locus conferring resistance to grapevine downy mildew. Theoretical and Applied Genetics 123: 43-53.

Bowen J, Mesarich C, Rees-George J, Cui W, Fitzgerald A, Win J, Plummer K, Templeton M, 2009. Candidate effector gene identification in the ascomycete fungal phytopathogen Venturia inaequalis by expressed sequence tag analysis. Molecular Plant Pathology 10: 431-448.

Brown M, Moore J, Fenn P, McNew RW, 1999. Evaluation of grape germplasm for downy mildew resistance. Fruit Varieties Journal 53: 22-29.

Cabral A, Stassen J, Seidl M, Bautor J, Parker J, van den Ackerveken G, 2011. Identification of Hyaloperonospora arabidopsidis transcript sequences expressed during infection reveals isolate-specific effectors. PLoS ONE 6: e19328.

Cadle-Davidson L, 2008. Variation within and between Vitis spp. for foliar resistance to the downy mildew pathogen Plasmopara viticola. Plant Disease 92: 1577-1584.

Catanzariti AM, Dodds PN, Lawrence GJ, Ayliffe MA, Ellis JG, 2006. Haustorially expressed secreted proteins from flax rust are highly enriched for avirulence elicitors. Plant Cell 18: 243-256.

Cramer R, La Rota C, Cho Y, Thon M, Craven K, Knudson D, Mitchell T, Lawrence C, 2006. Bioinformatic analysis of expressed sequence tags derived from a compatible Alternaria brassicicola-Brassica oleracea interaction. Molecular Plant Pathology 7: 113-124.

van Damme E, Barre A, Rouge P, Peumans W, 2004. Cytoplasmic/nuclear plant lectins: a new story. Trends in Plant Science 9: 484-489.

Denzer H, Staudt G, Schlosser E, 1995. The behavior of Plasmopara viticola on resistant and susceptible grapevine varieties. Vitis 34: 113-117.

Diez-Navajas AM, Greif C, Poutaraud A, Merdinoglu D, 2007. Two simplified fluorescent staining techniques to observe infection structures of the oomycete Plasmopara viticola in grapevine leaf tissues. Micron 38: 680-683.

Dong S, Qutob D, Tedman-Jones J, Kuflu K, Wang Y, Tyler B, Gijzen M, 2009. The Phytophthora sojae avirulence locus Aur3c encodes a multi-copy RXLR effector with sequence polymorphisms among pathogen strains. PLOS ONE 4: e5556.

Emanuelsson O, Brunak S, von Heijne G, Nielsen H, 2007. Locating proteins in the cell using TargetP, SignalP and related tools. Nature Protocols 2: 953-971. 
Fujita T, 2002. Evolution of the lectin-complement pathway and its role in innate immunity. Nature Reviews Immunology 2: 346-353.

Gessler C, Pertot I, Perazzolli M, 2011. Plasmopara viticola: a review of knowledge on downy mildew of grapevine and effective disease management. Phytopathologia Mediterranea 50: 3-44.

Haas B, Kamoun S, Zody M, Jiang R, Handsaker R, et al., 2009. Genome sequence and analysis of the Irish potato famine pathogen Phytophthora infestans. Nature 461: 393-398.

Hama H, Schnieders E, Thorner J, Takemoto J, DeWald D, 1999. Direct involvement of phosphatidylinositol 4-phosphate in secretion in the yeast Saccharomyces cerevisiae. Journal of Biological Chemistry 274: 34294-34300.

Hamshou M, Van Damme E, Smagghe G, 2010. Entomotoxic effects of fungal lectin from Rhizoctonia solani towards Spodoptera littoralis. Fungal Biology 114: 34-40.

Hoegger P, Kilaru S, James T, Thacker J, Kues U, 2006. Phylogenetic comparison and classification of laccase and related multicopper oxidase protein sequences. FEBS Journal 273: 2308-2326.

de Hoff P, Brill L, Hirsch A, 2009. Plant lectins: the ties that bind in root symbiosis and plant defense. Molecular Genetics and $\mathrm{Ge}$ nomics 282: 1-15.

Huitema E, Vleeshouwers V, Cakir C, Kamoun S, Govers F, 2005. Differences in intensity and specificity of hypersensitive response induction in Nicotiana spp. by INF1, INF2A, and INF2B of Phytophthora infestans. Molecular Plant-Microbe Interactions 18: 183-193.

Hunter S, Apweiler R, Attwood T, Bairoch A, Bateman A, Binns D, Bork P, Das U, Daugherty L, Duquenne L, Finn R, Gough J, Haft D, Hulo N, Kahn D, Kelly E, Laugraud A, Letunic I, Lonsdale D, Lopez R, Madera M, Maslen J, McAnulla C, McDowall J, Mistry J, Mitchell A, Mulder N, Natale D, Orengo C, Quinn A, Selengut J, Sigrist C, Thimma M, Thomas P, Valentin F, Wilson D, Wu C, Yeats C, 2009. InterPro: the integrative protein signature database. Nucleic Acids Research 37: D211-D215.

Jaillon O, Aury JM, Noel B, Policriti A, Clepet C, et al., 2007. The grapevine genome sequence suggests ancestral hexaploidization in major angiosperm phyla. Nature 449: 463-468.

Jenkins J, Pickersgill R, 2001. The architecture of parallel betahelices and related folds. Progress in Biophysics \& Molecular Biology 77: 111-175.

Jiang R, Tyler B, Whisson S, Hardham A, Govers F, 2006. Ancient origin of elicitin gene clusters in Phytophthora genomes. Molecular Biology and Evolution 23: 338-351.

de Jonge R, Bolton MD, Thomma BP, 2011. How filamentous pathogens co-opt plants: the ins and outs of fungal effectors. Current Opinion in Plant Biology 14: 400-406.

Judelson HS, Blanco FA, 2005. The spores of Phytophthora: weapons of the plant destroyer. Nature Reviews Microbiology 3: 47-58.

Kamoun S, 2006. A catalogue of the effector secretome of plant pathogenic oomycetes. Annual Review of Phytopathology 44: 41-60.

Kortekamp A, Zyprian E, 2003. Characterization of Plasmopararesistance in grapevine using in vitro plants. Journal of Plant Physiology 160: 1393-1400.

Lederkremer G, 2009. Glycoprotein folding, quality control and ER-associated degradation. Current Opinion in Structural Biology 19: $515-523$.

Marguerit E, Boury C, Manicki A, Donnart M, Butterlin G, Nemorin A, Wiedemann-Merdinoglu S, Merdinoglu D, Ollat N, Decroocq S, 2009. Genetic dissection of sex determinism, inflorescence morphology and downy mildew resistance in grapevine. Theoretical and Applied Genetics 118: 1261-1278.

Marino R, Sevini F, Madini A, Vecchione A, Pertot M, Serra A, Versini G, Velasco R, Grando M, 2003. QTL mapping for disease resistance and fruit quality in grape. Acta Horticulturae $527-533$.
Merdinoglu D, Wiedemann-Merdinoglu S, Coste P, Dumas V, Haetty S, Butterlin G, Greif C, 2003. Genetic analysis of downy mildew resistance derived from Muscadinia rotundifolia. Acta Horticulturae 451-456.

Michell R, 2008. Inositol derivatives: evolution and functions. Nature Reviews Molecular Cell Biology 9: 151-161.

Michelmore RW, 2003. The impact zone: genomics and breeding for durable disease resistance. Current Opinion in Plant Biology 6: 397-404.

Nakamura K, Go N, 2005. Function and molecular evolution of multicopper blue proteins. Cellular and Molecular Life Sciences 62: 2050-2066.

Odorizzi G, Babst M, Emr S, 2000. Phosphoinositide signaling and the regulation of membrane trafficking in yeast. Trends in Biochemical Sciences 25: 229-235.

Peressotti E, Wiedemann-Merdinoglu S, Delmotte F, Bellin D, Di Gaspero G, Testolin R, Merdinoglu D, Mestre P, 2010. Breakdown of resistance to grapevine downy mildew upon limited deployment of a resistant variety. BMC Plant Biology 10: 147.

Pezet R, Gindro K, Viret O, Richter H, 2004. Effects of resveratrol, viniferins and pterostilbene on Plasmopara viticola zoospore mobility and disease development. Vitis 43: 145-148.

Pezet R, Pont V, Hoangvan K, 1991. Evidence for oxidative detoxification of pterostilbene and resveratrol by a laccase-like stilbene oxidase produced by Botrytis cinerea. Physiological and Molecular Plant Pathology 39: 441-450.

Polesani M, Desario F, Ferrarini A, Zamboni A, Pezzotti M, Kortekamp A, Polverari A, 2008. cDNA-AFLP analysis of plant and pathogen genes expressed in grapevine infected with Plasmopara viticola. BMC Genomics 9: 142.

Riemann M, Buche C, Kassemeyer HH, Nick P, 2002. Cytoskeletal responses during early development of the downy mildew of grapevine (Plasmopara viticola). Protoplasma 219: 13-22.

Santagata S, Boggon T, Baird C, Gomez C, Zhao J, Shan W, Myszka D, Shapiro L, 2001. G-protein signaling through tubby proteins. Science 292: 2041-2050.

Schmidlin L, Poutaraud A, Claudel P, Mestre P, Prado E, SantosRosa M, Wiedemann-Merdinoglu S, Karst F, Merdinoglu D, Hugueney P, 2008. A stress-inducible resveratrol O-methyltransferase involved in the biosynthesis of pterostilbene in grapevine. Plant Physiology 148: 1630-1639.

Schornack S, Huitema E, Cano L, Bozkurt T, Oliva R, van Damme M, Schwizer S, Raffaele S, Chaparro-Garcia A, Farrer R, Segretin M, Bos J, Haas B, Zody M, Nusbaum C, Win J, Thines M, Kamoun S, 2009. Ten things to know about oomycete effectors. Molecular Plant Pathology 10: 795-803.

Staden R, 1996. The Staden sequence analysis package. Molecular Biotechnology 5: 233-241.

Stassen JH, van den Ackerveken GV, 2011. How do oomycete effectors interfere with plant life? Current Opinion in Plant Biology 14: 407-414

Staudt G, Kassemeyer H, 1995. Evaluation of downy mildew resistance in various accessions of wild Vitis species. Vitis 34 : 225-228.

Tian M, Huitema E, da Cunha L, Torto-Alalibo T, Kamoun S, 2004. A Kazal-like extracellular serine protease inhibitor from Phytophthora infestans targets the tomato pathogenesis-related protease P69B. Journal of Biological Chemistry 279: 26370-26377.

Torto-Alalibo T, Tripathy S, Smith B, Arredondo F, Zhou L, Li H, Chibucos M, Qutob D, Gijzen M, Mao C, Sobral B, Waugh M, Mitchell T, Dean R, Tyler B, 2007. Expressed sequence tags from Phytophthora sojae reveal genes specific to development and infection. Molecular Plant-Microbe Interactions 20: 781-793.

Vaara M, 1992. 8 bacterial proteins, including UDP-Nacetylglucosamine acyltransferase (LPXA) and 3 other transferases of Escherichia coli, consist of a 6-residue periodicity theme. FEMS Microbiology Letters 97: 249-254. 
Viennot-Bourgin G, 1949. Les champignons parasites de plantes cultivées. Masson \& Cie, Paris.

Vleeshouwers V, Rietman H, Krenek P, Champouret N, Young C, Oh S, Wang M, Bouwmeester K, Vosman B, Visser R, Jacobsen E, Govers F, Kamoun S, Van der Vossen E, 2008. Effector genomics accelerates discovery and functional profiling of potato disease resistance and Phytophthora infestans avirulence genes. PLoS ONE 3: e2875.

Welter L, Gokturk-Baydar N, Akkurt M, Maul E, Eibach R, Topfer R, Zyprian E, 2007. Genetic mapping and localization of quantitative trait loci affecting fungal disease resistance and leaf morphology in grapevine (Vitis vinifera L). Molecular Breeding 20: 359-374.

Wu J, Zhang Y, Zhang H, Huang H, Folta K, Lu J, 2010. Whole genome wide expression profiles of Vitis amurensis grape responding to downy mildew by using Solexa sequencing technology. BMC Plant Biology 10: 234.

Zeng Y, Yang T, 2002. RNA isolation from highly viscous samples rich in polyphenols and polysaccharides. Plant Molecular Biology Reporter 20 417a-417e. 